\title{
Chemokine receptor CCR5 mediates resistance to West Nile Virus infection in mouse and man Philip Murphy*
}

Address: National Institute of Allergy and Infectious Diseases, National Institutes of Health, Bethesda, Maryland 20892, USA

* Corresponding author

from 2006 International Meeting of The Institute of Human Virology

Baltimore, USA. 17-21 November, 2006

Published: 21 December 2006

Retrovirology 2006, 3(Suppl I):S2 doi:I0.1 I86/1742-4690-3-SI-S2

(C) 2006 Murphy; licensee BioMed Central Ltd.

West Nile Virus (WNV) is a re-emerging pathogen and a well-known agent of fatal encephalitis in several species, including mouse and man; however immunopathogenic mechanisms are poorly understood. We found that WNV induced upregulation of the chemokine receptor/HIV coreceptor CCR5 and its ligand CCL5 in mouse brain, as well as influx of effector leukocytes, including CD4+ and CD8+ T cells, NK cells, and macrophages, of which 25\% expressed CCR5. Infection of CCR5-/- mice was rapidly and uniformly fatal, whereas the majority of CCR $5+/+$ mice survived. Consistent with this, CCR5 32 homozygotes were overrepresented in three of three cohorts of symptomatic WNV seropositive individuals compared to control populations. And were more likely to have a fatal outcome. CCR5 appears to mediate resistance to fatal WNV infection in mouse and man by coordinating leukocyte recruitment to the infected brain. Thus CCR5 32 homozygosity is a strong genetic risk factor for symptomatic WNV infection in man. The results suggest that therapeutic blockade of CCR5 may carry increased risk of symptomatic and possibly fatal outcome should individuals become infected with WNV. 